Research Paper

\title{
MEOX1 Promotes Tumor Progression and Predicts Poor Prognosis in Human Non-Small-Cell Lung Cancer
}

\author{
Lichao Sun $^{1}$, Hebao Yuan², Joseph Burnett², Mari Gasparyan², Yuan Zhang1, Feng Zhang1, Zhihua Yang1, \\ Yuliang Ran ${ }^{1}$, Duxin Sun ${ }^{2}$ \\ 1. State Key Laboratory of Molecular Oncology, Cancer Hospital, Chinese Academy of Medical Sciences, Peking Union Medical College, Beijing, 100021, P. R. \\ China \\ 2. Department of Pharmaceutical Sciences, University of Michigan, Ann Arbor, MI 48109
}

$\square$ Corresponding authors: Lichao Sun, PhD, State Key Laboratory of Molecular Oncology, Cancer Hospital, Chinese Academy of Medical Sciences, Peking Union Medical College, Beijing, 100021, P. R. China. Email: sunlichao@cicams.ac.cn and Duxin Sun, PhD, Department of Pharmaceutical Sciences, University of Michigan, Ann Arbor, MI 48109. Email: duxins@umich.edu

(c) Ivyspring International Publisher. This is an open access article distributed under the terms of the Creative Commons Attribution (CC BY-NC) license (https://creativecommons.org/licenses/by-nc/4.0/). See http://ivyspring.com/terms for full terms and conditions.

Received: 2018.05.31; Accepted: 2018.11.26; Published: 2019.01.01

\begin{abstract}
Background: MEOX 1 is a homeobox transcriptional factor, and plays essential roles in regulating somite development. Our previous study indicated that MEOXI is a critical molecular target in mesenchymal-like cancer cells in PTEN-deficient Trastuzumab resistant breast cancer. Despite the potential implication of MEOX1 for the cancer progression, no previous studies examined its level and clinical significance in lung cancer tissues. In this study, we aimed to detect the MEOXI expression and correlate its level with clinical outcome in non-small-cell lung cancer patients (NSCLC).

Methods: MEOX1 gene expression in lung cancer was examined by using the Oncomine database. MEOXI protein levels were evaluated by IHC using the corresponding primary antibody on two different commercial lung cancer tissue arrays. siRNA knockdown was used to elucidate the function of MEOX1.

Results: Analysis of the Oncomine datasets identified that an elevation of MEOX1 in gene amplification in lung cancer tissues in comparison to normal lung tissues. Immunohistochemistical analysis demonstrated that MEOX1 was localized predominantly in the nucleus, and positive rate was $67.3 \%(111 / 165)$ in NSCLC samples. Statistical analysis revealed high levels of MEOX1 significantly correlated with Lymph Node Metastasis and Stage. Kaplan-Meier survival analysis showed that high levels of MEOXI were significantly associated with unfavorable survival in NSCLC patients, and MEOX 1 nucleus staining had worse survival, than did patients with overall expression in lung squamous cell carcinoma patients. Multivariate Cox's regression analysis found that MEOXI was an independent poor prognostic predictor for patients with NSCLC. Silencing of MEOXI by specific SiRNA significantly inhibited H460 and H1299 cell proliferation and sphere formation in serum-free medium.

Conclusions: Our results firstly indentified that high levels of MEOXI especially nuclear staining was an independent prognostic factor for NSCLC, and it served a essential roles in the regulation of cell proliferation and colony formation in vitro. It may represent a potential target for the NSCLC treatment.
\end{abstract}

Key words: lung cancer, MEOX1, prognosis

\section{Introduction}

Lung cancer is one of the most common cancers with highest mortality rate in China[1]. Comparing with other pathological type, Non-small-cell lung cancer (NSCLC) accounts for approximately $80-85 \%$ 
of lung cancer cases[2]. Despite great improvements in treatment over recent decades, the outcome of patients has not significantly improved. Therefore, it is necessary to identify the novel therapeutic targets for lung cancer treatment.

MEOX1 is a critical homeobox transcriptional factor, which could affect the somite formation[3]. Several studies have proved that dysregulation of MEOX1 is associated with cancer progression. In ovarian cancer, MEOX1, as a PBX1 cofactor in, mediates the cellular growth signal[4]. In Triple-negative breast cancer (TNBC), MEOX1 was overexpressed in mesenchymal stem-like subtype cancer cells, which were sensitive to NVP-BEZ235 and dasatinib treatment [5]. Our previous study identified that MEOX1 was overexpressed in breast cancer tissues and played key roles in developmement of trastuzumabresistance and epithelial to mesenchymal transtion. In addition, down-regulation of MEOX1 could decrease mammosphere and colony formation in vitro, and inhibit the tumor growth and breast cancer stem cell frequency in vivo[6]. Although MEOX1 might play an important role in carcinogenesis, no previous studies investigated its expression, clinical significance and function in lung cancer.

In this study, we not only demonstrated that MEOX1 was significantly elevated and closely associated with poor prognosis of NSCLC patients, but also regulated cell proliferation and sphere formation in vitro for the first time.

\section{Materials and methods}

\section{Analysis of Oncomine data}

Oncomine was used for the analysis of MEOX1 level in lung cancer (http://www.oncomine.org). Gene expression was evaluated by comparing lung cancer and normal patient datasets. The analyzing procedure was performed according to previous studies[7].

\section{Clinical samples and evaluation of immunostaining}

Two different commercial lung cancer tissue arrays were constructed by Shanghai Biochip Co. Ltd. as described[8]. Briefly, lung adenocarcinoma tissue array contains 90 cases of patients, and lung squamous cell carcinoma tissue array contains 75 cases respectively. Both of them contained the correspondent adjacent normal tissues. For all the specimens, clinicopathological factors and survival status were available.

The MEOX1 protein levels were evaluated by standard Avidin-biotin complex peroxidase immunohistochemical staining. Briefly, after deparaffinizatio- nin xylene and graded alcohols, heated antigen retrieval was done in citrate buffer $(10 \mathrm{mmol} / \mathrm{L} \mathrm{pH}$ 6.0) by water-bath kettle heating for $30 \mathrm{~min}$. Endogenous peroxidase was blocked in $0.3 \%$ hydrogen peroxide for $10 \mathrm{~min}$. Nonspecific binding was blocked by incubation in $10 \%$ normal animal serum for $10 \mathrm{~min}$. Sections were incubated at $4^{\circ} \mathrm{C}$ for $24 \mathrm{~h}$ with primary antibodies against MEOX1 (HPA045214, SigmaAldrich). We employed semi-quantitative scoring system in considering the staining intensity and the percentage of positive cancer cells, which has been widely accepted and used in previous studies[6,9]. Tissues with no or faint staining were rated as 0 , with moderate staining as 1 , with strong staining as 2 . The percentage of positive cells was scored as follows: $0(0$ to $25 \%), 1(25 \%-50 \%), 2(>50 \%)$. The results were determined using the following formula: overall score $=$ positive percentage score $\times$ intensity score. The staining were defined as negative (from 0 to 2 ), and positive $(>2)$.

\section{Knockdown by siRNA}

SiRNA targeting human MEOX1was purchased from Qiagen (validated FlexiTube siRNA). Lipofectamine ${ }^{\circledR}$ RNAiMAX was used to transfect cells. As a negative control, a non-targeting sequence siRNA was employed (Qiagen, 1027281). Knockdown at mRNA level was confirmed by real-time quantitative RTPCR. MEOX1 Primer: Forward primer, 5-GAAACCC CCACTCGGAAGG-3, Reverse primer, 5-GGGTGCT GCTCAGTGAAGAT-3.

\section{MTS Cell Proliferation Assay}

Cancer cells were seeded at a density of 2,000 cells per well in 96-well plates. Cell growth was determined by MTS assay according to manufacturer's instruction by measuring the absorbance at 490 $\mathrm{nm}$ on a Synergy 2 plate reader (Biotek).

\section{Mammosphere Formation Assay}

Mammosphere culture was done according to MammoCultt ${ }^{\mathrm{TM}}$ Human Medium Kit (05620, STEM CELL Technologies Inc.) supplemented with Heparin and Hydrocortisone. Single cells were plated in six-well ultralow attachment plates (Corning) at a density of 500 cells/ml. After 14 days of culture, the number of mammospheres was counted on a Nikon Eclipse TE2000-S microscope and the photos were acquired with MetaMorph 7.6.0.0.

\section{Statistical Analysis}

The SPSS 15 software package (SPSS, Inc., Chicago, IL) was used for statistical analysis. The association between MEOX1 expression and clinicopathologic features was analyzed by using $x^{2}$-test or two-sided t-test as appropriate. Kaplan-Meier analysis 
and log-rank test were used to evaluate the overall survival (OS) of patients with lung cancer according to the expression of MEOX1. Cox's proportional hazards regression model was used for multivariate analysis of survival in lung cancer patients. All comparisons were two-tailed, and p-value of $<0.05$ was considered statistically significant.

\section{Results}

\section{Gene expression of MEOX1 in lung cancer}

By using Oncomine datasets, we investigated the MEOX1 gene expression in lung cancer. The results showed that MEOX1 gene copy number in lung cancer tissues was higher than in normal tissues in TCGA and Weiss Lung datasets (Figure. 1A, 1B). Moreover, up-regulation of MEOX1 was also found in Squamous cell lung carcinoma with distant metastasis (M1 stage) in compared with ones without distant metastasis (M0 stage) in TCGA dataset (Figure 1C). Taken together, these data indicated that MEOX1 was highly expressed in lung cancer tissues especially in NSCLC, which might play essential roles in lung cancer progression.

A

MEOX1 Copy Number in TCGA Lung 2

Grouped by Cancer and Normal Type

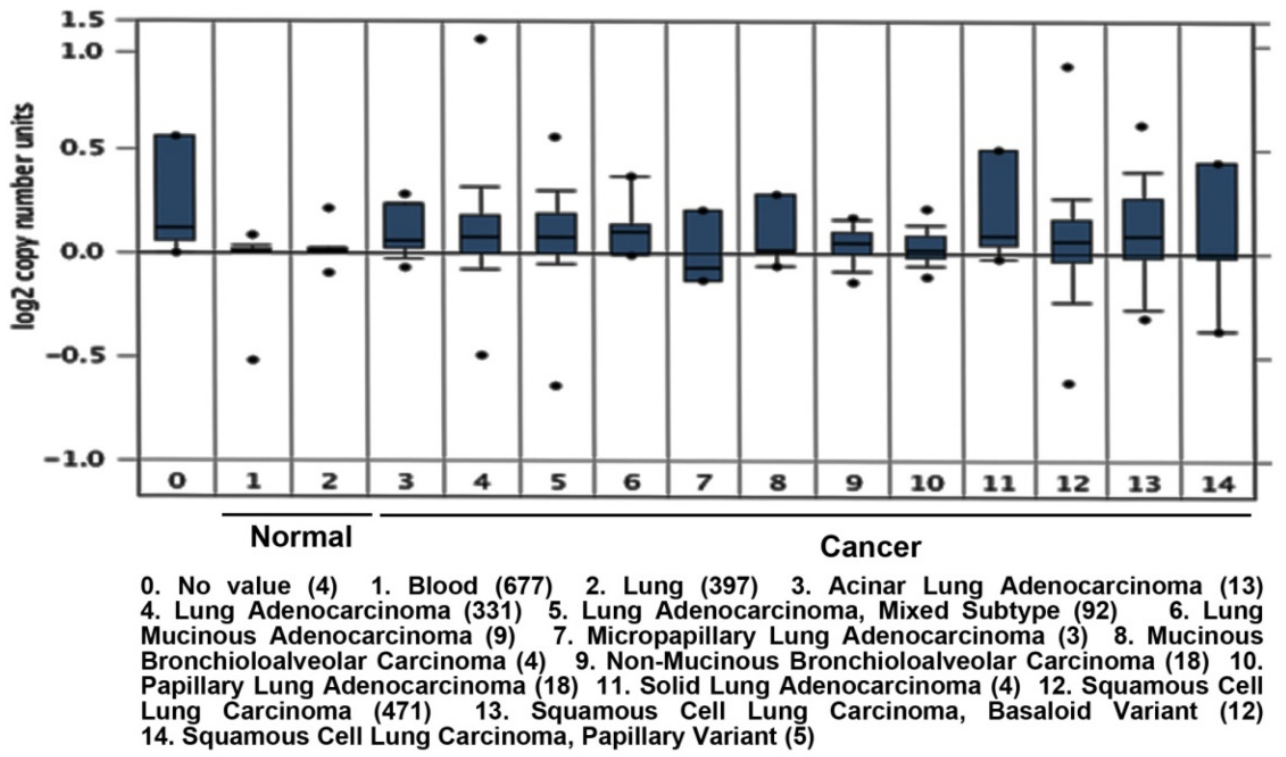

B

MEOX1 Copy Number in Weiss Lung Grouped by Cancer and Normal Type

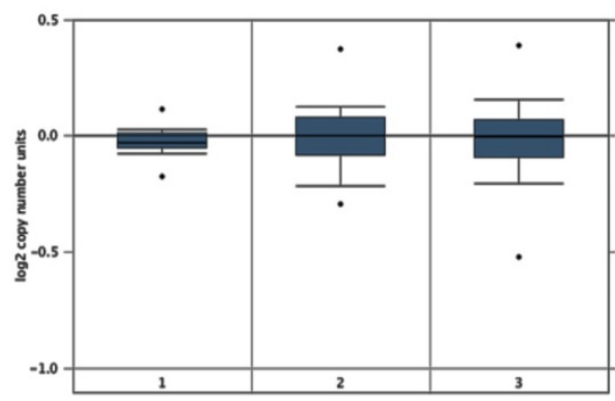

1. Lung (59) 2. Lung Adenocarcinoma (77) 3.

Squamous Cell Lung Carcinoma (155)
C MEOX1 Expression in TCGA Lung Grouped by M Stage (Squamous Cell Lung Carcinoma)

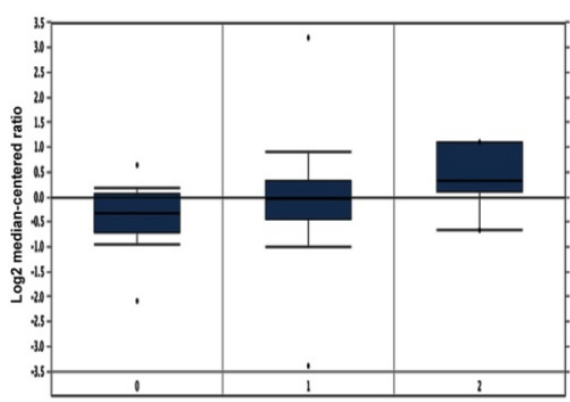

0. No value (41) 1. M0 (142) 2. M1+ (4)

Figure 1. MEOX1 mRNA or DNA expression in human lung cancers using the Oncomine database. A. MEOXI gene copy number in human lung cancers vs. normal tissues in TCGA. B. MEOX1 gene copy number in human lung cancers vs. normal tissues in Weiss datasets. C. MEOXI mRNA expression in Squamous cell lung carcinoma grouped by $M$ stage. 
A
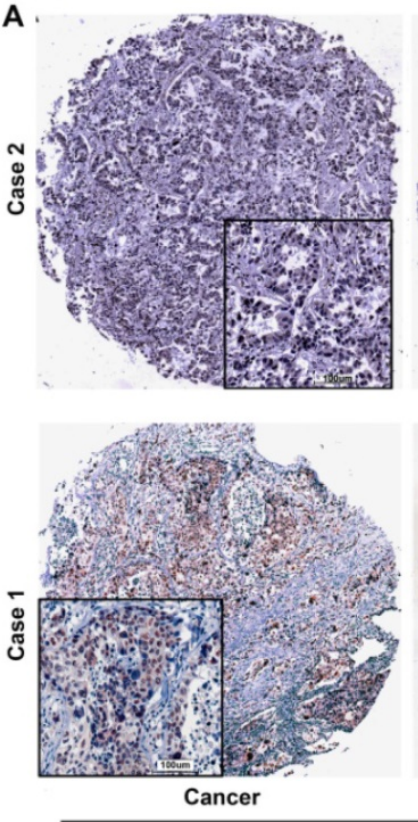
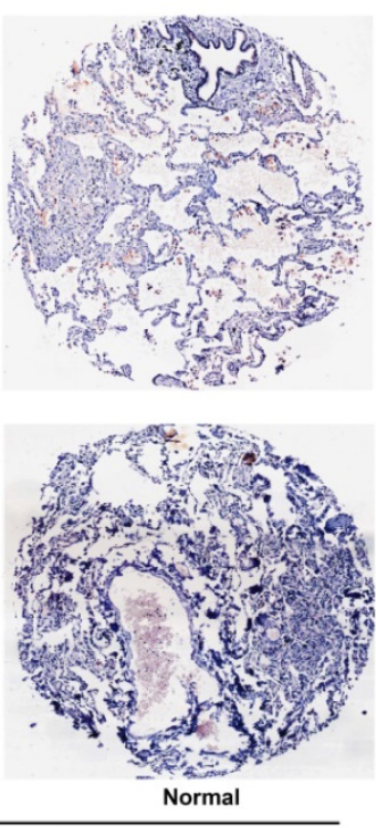

B
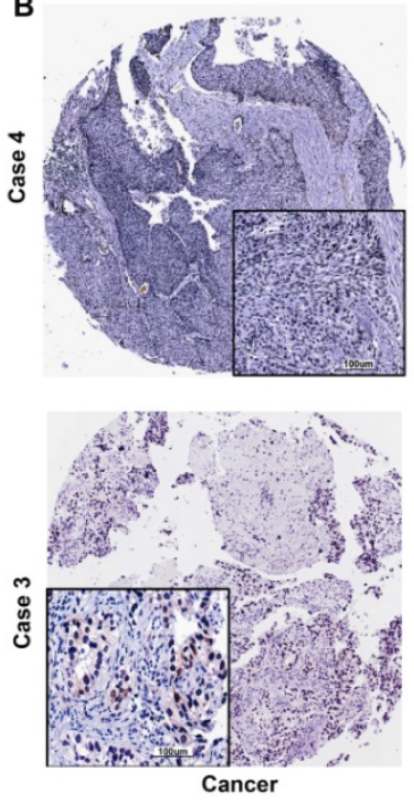

Lung squamous cell carcinoma
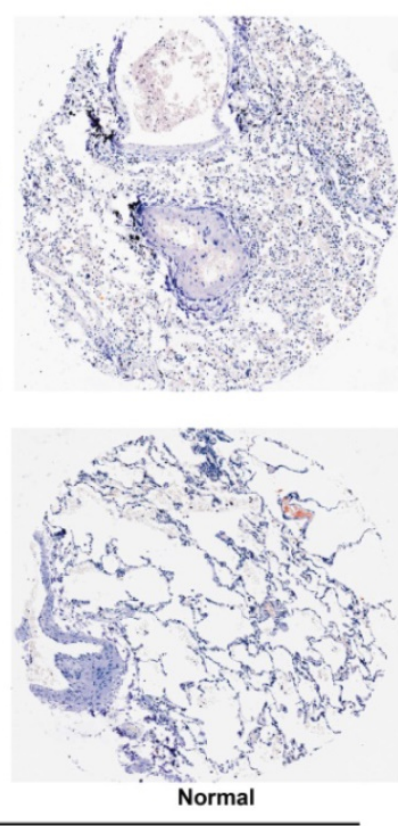

lung adenocarcinoma Figure 2. MEOX1 expression in lung cancer tissues is determin
tissues. B. Representative staining of squamous cell lung carcinoma tissues.

\section{Over-expression of MEOX1 protein in NSCLC tissues by immunohistochemistry analysis}

We evaluated the expression of the MEOX1 in Human NSCLC tissue arrays by immunohistochemistry. The results demonstrated that MEOX1 was positive in $111 / 165$ primary tumors $(67.3 \%)$, but there was no or weak staining of adjacent normal tissues. Moreover, MEOX1 was predominantly localized to the nucleus (Figure. 2). Statistical analysis revealed that whether cytoplasmic or nuclear MEOX1 staining was significantly associated with Lymph Node metastasis and Stage. Furthermore, MEOX1 expression was more frequent in adenocarcinoma than squamous cell carcinoma $(P=0.000)$. However, no statistically significant correlations were identified between the expression of MEOX1 and other clinicopathologic characteristics (Table 1).

\section{MEOXI expression level correlated with poor prognosis of NSCLC}

Kaplan-Meier analysis showed that the overall survival time of lung cancer patients with high levels of MEOX1 was markedly shorter than those with MEOX1-negative expression (Figure. 3A, 3B). Further analysis indicated that MEOX1 nucleus staining had worse survival, than did patients with overall expression in squamous cell lung cancer patients (Figure. 3C). Then, we performed Cox multivariate regression model to test the independent value of each variable predicting overall survival. The results revealed that MEOX1 nuclear staining level
( $\mathrm{HR}=3.304 ; 95 \% \mathrm{CI}: 2.115-5.161 ; \mathrm{P}=0.000)$ and Stage ( $\mathrm{HR}=1.750 ; \quad 95 \% \quad \mathrm{CI}: \quad 1.030-2.972 ; \quad \mathrm{P}=0.038)$ were statistically independent predictive factors of poorer prognosis for lung cancer (Table 2).

Table 1. Correlation between MEOXI expression and clinicopathological parameters in 165 lung cancer cases

\begin{tabular}{|c|c|c|c|c|c|c|}
\hline & \multicolumn{3}{|c|}{ Total MEOX1 staining } & \multicolumn{3}{|c|}{ MEOX1 nuclear staining } \\
\hline & negative & positive & $\begin{array}{l}\mathrm{p}- \\
\text { value }\end{array}$ & negative & positive & $\begin{array}{l}\text { p-val } \\
\text { ue }\end{array}$ \\
\hline $\begin{array}{l}\text { Gender (Male: } \\
\text { Female) }\end{array}$ & $43: 11$ & $77: 34$ & 0.165 & 59:17 & $61: 28$ & 0.191 \\
\hline Age & $62.9 \pm 8.0$ & $63.2 \pm 10.2$ & 0.836 & $62.9 \pm 9.0$ & $63.3 \pm 10.0$ & 0.802 \\
\hline Type & & & 0.000 & & & 0.000 \\
\hline $\begin{array}{l}\text { Squamous cell } \\
\text { carcinoma }\end{array}$ & 35 & 40 & & 47 & 28 & \\
\hline $\begin{array}{l}\text { Adenocarcinoma } \\
\text { carcinoma }\end{array}$ & 19 & 71 & & 29 & 61 & \\
\hline Tumor size(cm) & & & 0.902 & & & 0.420 \\
\hline$<5$ & 37 & 75 & & 54 & 58 & \\
\hline$>5$ & 17 & 36 & & 22 & 31 & \\
\hline \multicolumn{2}{|c|}{ Depth of invasion } & & 0.801 & & & 0.833 \\
\hline $\mathrm{T} 1+\mathrm{T} 2$ & 45 & 95 & & 64 & 76 & \\
\hline $\mathrm{T} 3+\mathrm{T} 4$ & 9 & 16 & & 12 & 13 & \\
\hline \multicolumn{2}{|c|}{ Lymph node involvement } & & 0.001 & & & 0.000 \\
\hline No & 37 & 45 & & 49 & 33 & \\
\hline $\mathrm{N} 1+\mathrm{N} 2$ & 17 & 66 & & 27 & 56 & \\
\hline \multicolumn{2}{|c|}{ Distant metastasis } & & 0.321 & & & 0.910 \\
\hline M0 & 54 & 109 & & 75 & 88 & \\
\hline M1 & 0 & 2 & & 1 & 1 & \\
\hline Grade & & & 0.725 & & & 0.566 \\
\hline I+II & 40 & 85 & & 56 & 69 & \\
\hline III+IV & 14 & 26 & & 20 & 20 & \\
\hline Stage & & & 0.008 & & & 0.001 \\
\hline I+II & 38 & 54 & & 53 & 39 & \\
\hline III+IV & 16 & 57 & & 23 & 50 & \\
\hline
\end{tabular}


Table 2. Multivariate analysis of Cox Proportional Hazards Model for lung cancer

\begin{tabular}{lllllllll}
\hline Characteristics & B & SE & Wald & df & Sig. & $\begin{array}{l}\text { Exp } \\
\text { (B) }\end{array}$ & $\begin{array}{l}\text { 95.0\% CI for } \\
\text { Exp(B) }\end{array}$ \\
\hline $\begin{array}{l}\text { MEOX1 nuclear } \\
\text { staining }\end{array}$ & 1.195 & 0.228 & 27.576 & 1.000 & $\mathbf{0 . 0 0 0}$ & 3.304 & 2.115 & 5.161 \\
Stage & & & & & & & & \\
Gender & 0.560 & 0.270 & 4.285 & 1.000 & $\mathbf{0 . 0 3 8}$ & 1.750 & 1.030 & 2.972 \\
$\begin{array}{l}\text { Tumor Size } \\
\begin{array}{l}\text { Depth of invasion } \\
\text { Lymph node }\end{array}\end{array}$ & -0.028 & 0.225 & 0.016 & 1.000 & 0.900 & 0.972 & 0.625 & 1.512 \\
metastasis & -0.080 & 0.217 & 0.676 & 1.000 & 0.411 & 1.196 & 0.781 & 1.830 \\
Distance metastasis & 0.268 & 0.269 & 0.993 & 1.000 & 0.319 & 1.307 & 0.772 & 2.215 \\
\hline
\end{tabular}

\section{MEOX1 silencing suppressed the self-renewal and proliferation of lung cancer cell in vitro}

To study the function of MEOX1 in lung cancer cell line, we employed siRNA knockdown to study the effect of MEOX1 on cell proliferation and self-renewal ability in vitro. Silencing of MEOX1 significantly reduced its mRNA as determined by quantitative real-time PCR. The results showed that inhibition of MEOX1 dramatically suppressed the mammosphere formation number by $89.1 \%$ and $79.6 \%$ in H1299 and H460 cells over 14 days. And, mammosphere size was significantly decreased as well (Figure 4A). MTS assay indicated that knockdown of MEOX1 in H1299 cells grew at a rate $71.2 \%$ slower than control cells. Consistently, inhibition of MEOX1 also attenuated lung cancer cell H460 proliferation, and the inhibitory rate was approximately $51.2 \%$ (Figure 4B). Together, these results showed that MEOX1 might play important roles in lung cancer progression.

\section{Discussion}

Lung cancer is a leading cause of cancer death worldwide[10]. Approximately, non-small cell lung cancer (NSCLC) accounts for $85 \%$ lung cases, which is typically classified into two subtypes including adenocarcinoma (AD) and squamous cell carcinoma (SCC) $[7,11]$. Although novel treatment strategies have been developed, the lung cancer death rate still remains high[12-14]. Therefore, it is necessary to identify other novel targets for clinical practice.
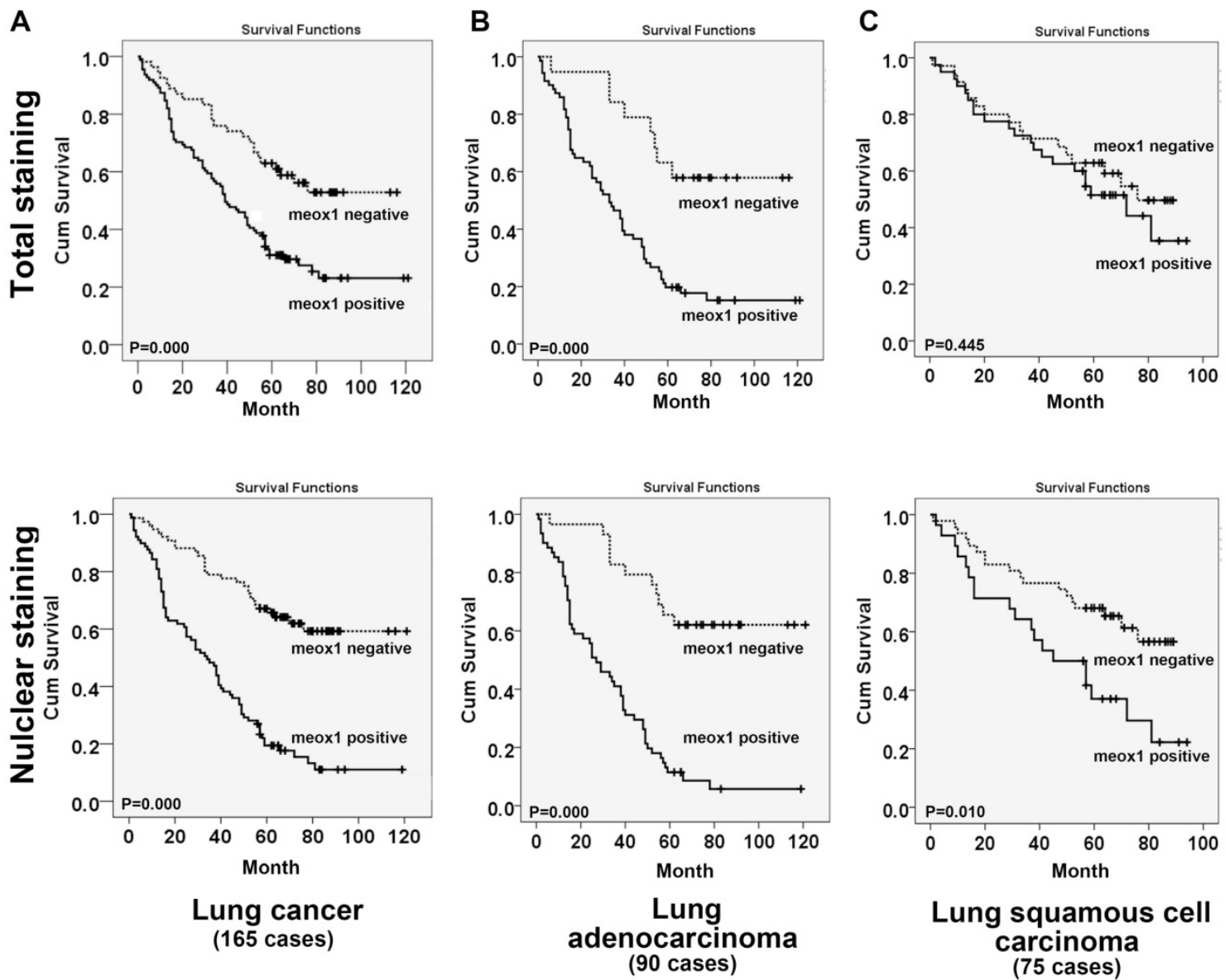

Figure 3. Survival curves for NSCLC cancer using the Kaplan-Meier method and the log-rank test. A. Overall survival curves for patients with negative MEOX1 expression (dot line) and patients with positive total or nuclear staining MEOX1 (solid line); B. Overall survival curves for lung adenocarcinoma patients with negative MEOXI expression (dot line) and patients with positive total or nuclear staining (solid line); C. Overall survival curves for lung squamous cell carcinoma patients with negative MEOX1 expression (dot line) and patients with positive total or nuclear staining MEOX1 (solid line). 
A
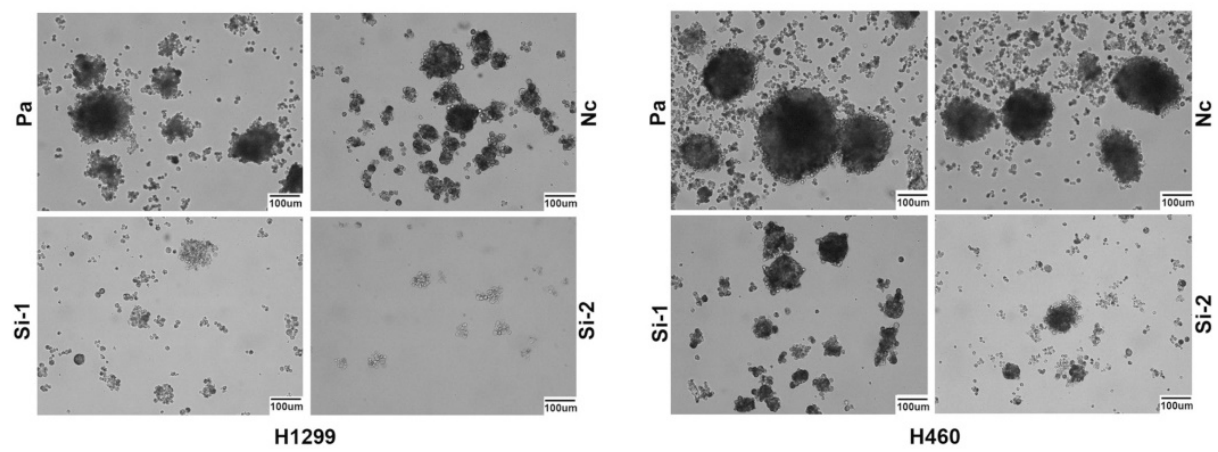

B
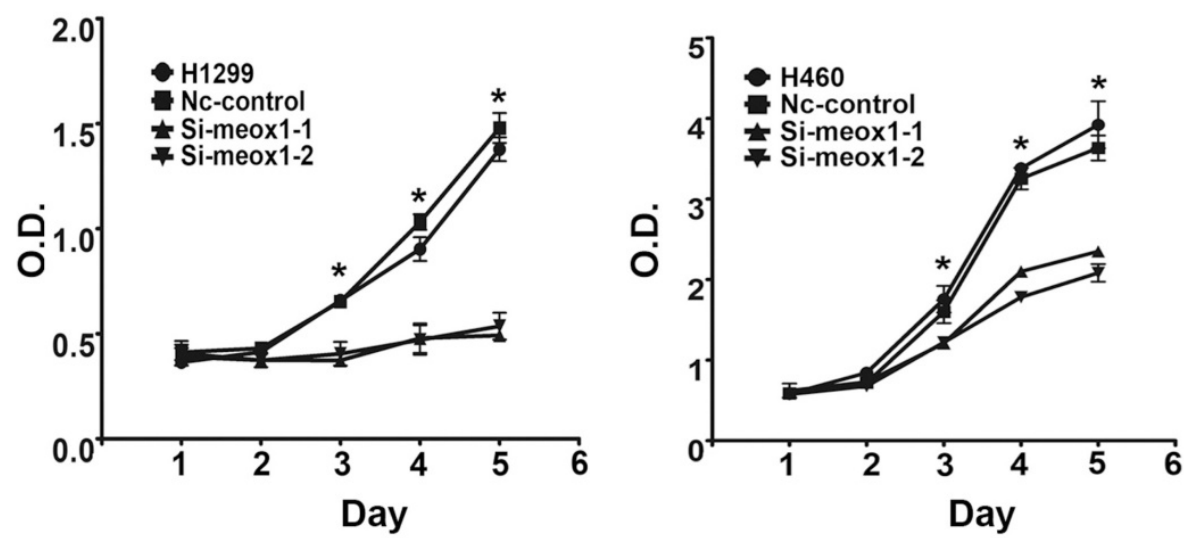

Figure 4. MEOX1 regulates lung cancer cell mammosphere formation and proliferation in vitro. A. Representative images of mammosphere formed 14 days after siRNA knockdown of MEOX1 in H1299 and H460 cells in comparison with parental or Nc-control cells. B. The growth of H1299 and H460 cell following MEOX1 knockdown as determined by MTS in comparison with parental or Nc-control cells.

MEOX1 is a critical homeobox gene, which is essential for the development of somite formation. Mutations in MEOX1 are associated with Klippel-Feil Syndrome[15]. It has been reported that aberrant expression of MEOX1 might involve in cancer progression. In ovarian cancer, MEOX1 was identified as a direct target gene of PBX1 involving in cancer cell growth[4]. Moreover, it has reported that MEOX1 was overexpressed in mesenchymal stem-like subtype cancer cells in TNBC [5]. Our previous study also demonstrated that MEOX1 might play key roles in mesenchymal-like breast cancer cell proliferation and BCSC self-renewal ability. Moreover, suppression of MEOX1 by sulforaphane (SF) was able to effectively inhibit bulk tumor volume and reduce frequency of BCSCs in an orthotopic xenograft mouse model of breast cancer Immuunohistochemistry assay showed that the expression of MEOX1 was specifically elevated in breast cancer tissues, and nuclear protein localization of MEOX1 was correlated with poorer overall survival, an advanced tumor stage, and the presence of lymph node metastasis[6]. Despite the potential implication of MEOX1 for the cancer progression, no previous studies have examined its level and clinical significance in lung cancer.
In this study, we firstly evaluated MEOX1 expression levels by using Oncomine datasets. We found that MEOX1 gene copy number was higher than in normal tissues in TCGA and Weiss Lung datasets. Interestingly, MEOX1 levels were higher in Squamous cell lung carcinoma with distant metastasis (M1 stage) than in ones without distant metastasis (M0 stage) in TCGA dataset. It is plausible that MEOX1 might contribute to lung cancer progression. Then, we detected MEOX1 protein level in human NSCLC tissue arrays by using IHC. The results showed that MEOX1 was highly expressed in NSCLC cancer tissues, with a positive rate of $67.3 \%$.

We do find the MEOX1 cytoplasmic staining in lung cancer tissues, and the positive rate was $16.0 \%$ $(12 / 75)$ in lung squamous cell carcinoma tissues and $11.1 \%(10 / 90)$ in lung adenocarcinoma tissues, respectively. As a transcriptional factor, we found that MEOX1 staining was predominantly localized to the nucleus. Clinical relevance analysis showed that positive expression of MEOX1 in NSCLC tissues was significantly correlated with Lymph Node Metastasis and Stage. Kaplan-Meier analysis indicated that lung cancer patients with high levels of MEOX1 had significantly shorter time than those with MEOX1- 
negative expression. Moreover, Cox multivariate regression model revealed that MEOX1 nuclear staining level and Stage were statistically independent predictive factors relating to poorer prognosis for lung cancer.

According to the MEOX1 level in lung cancer tissues and its clinical relevance, it is plausible that MEOX1 might promote tumor progression. Then we employed the SiRNA to knockdown its level in lung cancer cell line to assess its effects on cell growth in vitro. The results demonstrated that down-regulation of MEOX1 led to a significant reduction in lung cancer cell line H460 and H1299 proliferation and mammosphere formation in serum-free medium. In the future, we would further identify its downstream target genes, and determine the related molecular mechanism in lung cancer progression.

In conclusion, our study firstly demonstrated that over-expression of MEOX1 was significantly associated with poor survival in NSCLC. MEOX1 could be used as a potential novel target for human NSCLC.

\section{Acknowledgement}

Supported by grant from National Key R\&D Program of China (No: 2017YFC1308702), Beijing Nova Program (No: Z1511000003150121), Beijing Talents Fund (No:2015000021223ZK23), Beijing Gao Chuang Ji Hua (No.G02060050).

\section{Competing Interests}

The authors have declared that no competing interest exists.

\section{References}

1. Chen W, Zheng R, Baade PD, Zhang S, Zeng H, Bray F, et al. Cancer statistics in China, 2015. CA: a cancer journal for clinicians. 2016; 66: 115-32.

2. Siegel RL, Miller KD, Jemal A. Cancer Statistics, 2017. CA: a cancer journal for clinicians. 2017; 67: 7-30.

3. Mankoo BS, Skuntz S, Harrigan I, Grigorieva E, Candia A, Wright CVE, et al. The concerted action of Meox homeobox genes is required upstream of genetic pathways essential for the formation, patterning and differentiation of somites. Development. 2003; 130: 4655-64.

4. Thiaville MM, Stoeck A, Chen L, Wu RC, Magnani L, Oidtman J, et al. Identification of PBX1 target genes in cancer cells by global mapping of PBX1 binding sites. PloS one. 2012; 7: e36054.

5. Lehmann BD, Bauer JA, Chen X, Sanders ME, Chakravarthy AB, Shyr Y, et al. Identification of human triple-negative breast cancer subtypes and preclinical models for selection of targeted therapies. The Journal of Clinical Investigation. 2011; 121: 2750-67.

6. Sun L, Burnett J, Gasparyan M, Xu F, Jiang H, Lin CC, et al. Novel cancer stem cell targets during epithelial to mesenchymal transition in PTEN-deficient trastuzumab-resistant breast cancer. Oncotarget. 2016; 7: 51408-22.

7. Sun L, Wang Y, Yuan H, Burnett J, Pan J, Yang Z, et al. CPA4 is a Novel Diagnostic and Prognostic Marker for Human Non-Small-Cell Lung Cancer. Journal of Cancer. 2016; 7: 1197-204.

8. Chen L, Wei T, Si X, Wang Q, Li Y, Leng Y, et al. Lysine Acetyltransferase GCN5 Potentiates the Growth of Non-small Cell Lung Cancer via Promotion of E2F1, Cyclin D1, and Cyclin E1 Expression. The Journal of Biological Chemistry. 2013; 288: 14510-21.

9. Sun L, Hu H, Peng L, Zhou Z, Zhao X, Pan J, et al. P-Cadherin Promotes Liver Metastasis and Is Associated with Poor Prognosis in Colon Cancer. The American journal of pathology. 2011; 179: 380-90.

10. DeSantis CE, Lin CC, Mariotto AB, Siegel RL, Stein KD, Kramer JL, et al. Cancer treatment and survivorship statistics, 2014. CA Cancer J Clin. 2014; 64: 252-71.
11. Faruki H, Mayhew GM, Serody JS, Hayes DN, Perou CM, Lai-Goldman M. Lung Adenocarcinoma and Squamous Cell Carcinoma Gene Expression Subtypes Demonstrate Significant Differences in Tumor Immune Landscape. Journal of Thoracic Oncology. 2017;12: 943-953.

12. Singh $\mathrm{PK}$, Singh $\mathrm{H}$, Silakari O. Kinases inhibitors in lung cancer: From benchside to bedside. Biochimica et biophysica acta. 2016; 1866: 128-40.

13. Provencio M, Sánchez A, Garrido P, Valcárcel F. New Molecular Targeted Therapies Integrated With Radiation Therapy in Lung Cancer. Clinical Lung Cancer. 2010; 11: 91-7.

14. Sun L, Chen L, Sun L, Pan J, Yu L, Han L, et al. Functional Screen for Secreted Proteins by Monoclonal Antibody Library and Identification of Mac-2 Binding Protein (Mac-2BP) as a Potential Therapeutic Target and Biomarker for Lung Cancer. Molecular \& Cellular Proteomics. 2013; 12: 395-406.

15. Mohamed Jawahir Y, Faqeih E, Alsiddiky A, Alshammari Muneera J, Ibrahim Niema A, Alkuraya Fowzan S. Mutations in MEOX1, Encoding Mesenchyme Homeobox 1, Cause Klippel-Feil Anomaly. American Journal of Human Genetics. 2013; 92: 157-61. 